\title{
Physicians' attitudes toward the use of IoT medical devices as part of their practice
}

\author{
Dafni Biran Achituv, The Academic College of Tel Aviv-Yaffo, dafniba@gmail.com \\ Lior Haiman, restart Group, haimanlior@gmail.com
}

\begin{abstract}
The increasing number of patients using medical devices that are based on Internet of Things (IoT) technology presents physicians with a variety of challenges. The purpose of this exploratory research is to provide first insights into the way physicians perceive FDA approved IoT medical devices (IoT-MDs). A questionnaire was developed and improved after a pilot survey with the participation of 23 physicians. Data was collected from 126 physicians in 2014 and from another 50 in 2015, who answered the questionnaire, as well as from four physicians, who were interviewed. The combined results were analyzed, and a comparison between the two surveys was made. Results show that there is still not enough awareness and readiness for the use of IoT-MDs, and that there was no significant change in physicians' attitudes in 2015 compared to 2014. However, results show some differences between physicians who had previously been exposed to IoT technology and those who had not. The authors believe that IoTMDs generate data that is too raw for practical use, thereby limiting potential effectiveness. Applications that extract and highlight measured irregularities and that provide high-level, integrated information will increase physicians' openness to IoT-MDs, and will enable medical practice to be more efficient.
\end{abstract}

Keywords: Internet of Things, Healthcare, Patient-doctor Relationship, M-health, Impact of Medical Knowledge, Telehealth, Exposure to Technology.

\section{Introduction}

About twenty years after Ashton coined the phrase "Internet of Things", he explained how "in the real world, 'things' matter more than 'ideas"' (Ashton, 2009, p. 1). Indeed, over the past few years it has become impossible to avoid the buzzword Internet of Things (IoT). Major players like Google and Intel have started to invest heavily toward the realization of the concept. The IEEE forecast is 50 billion connections between IoT devices by 2020 ("Top 10 communications technology," 2015).

An IoT device is defined as any device capable of communicating with any other distinct devices via the Internet. These smart devices hold digital identities which allow them to interact and communicate amongst themselves and with the environment, while reacting autonomously to the physical world (Santucci, 2010). Integrating smart devices in smart environments allows for personal, social and spatial communications that are unencumbered by the manual handling that dominated the pre-IoT world (Laranjo, Macedo, \& Santos, 2012). In the IoT world, every day-today object has the potential to interpret and respond to its environment. It may do this using Radio-Frequency Identification (RFID) or sensors. This ability, coupled with network access, 
gives the IoT the potential to revolutionize many industries, with the medical industry at the top of the list (Fundación de la Innovación Bankinter, 2011). Moreover, the IoT is probably the most impacting socio-technological trend currently influencing health services (Couturier, Sola, Scarso Borioli, \& Raiciu, 2012).

Ubiquitous healthcare has been envisioned for the past two decades. IoT gives a perfect platform to realize this vision (Santucci, 2010). For instance, RFID-supporting devices can be implanted in patients suffering from conditions that necessitate close monitoring. Each device's identification is paired with a patient, and gives doctors instant access to the patient's medical history. More extensive implementation of RFID technology could advance and streamline health services, such as consumption of medication by patients (Laranjo et al., 2012). However, even with all the evident benefits of IoT technologies, the prospect of their implementation on all levels of society cannot be taken for granted. IoT devices face many challenges on their way to ensuring widespread use. Although a comprehensive analysis of these challenges is beyond the scope of this study, several of the major issues are worth mentioning. The first of these is information security. Ubiquitous, public wireless communication exposes personal information, making non-encrypted data vulnerable (Whitmore, Agarwal, \& $\mathrm{Da} \mathrm{Xu}, 2015$ ). Hackers may attack these systems in many ways, including for example network shutdown, insertion of false information, and stealing personal information (Gubbi, Buyya, Marusic, \& Palaniswami, 2013). Encryption methods are the first line of defence in providing data confidentiality. Identity management, and true user identity verification in particular, are also expected to reduce potential damage and improve product reliability. However, it is necessary to continually track changes and developments in the field of attack technologies.

Another significant challenge for IoT adoption is the enormous amounts of data that are collected. Massive data generation by a device, the need for real-time uploads, and the extended use of the device result in enormous growth of data volumes expected every year. According to Gantz, and Reinsel (2012), by 2020, the volume of information will reach 40 trillion gigabytes, $40 \%$ of which will be either be processed or, stored in the cloud. This forecast raises questions about data management: how to manage and optimize the information system in an efficient and cost effective manner? (Phan, 2013).

A medical device is:

An instrument, apparatus, implement, machine, contrivance, implant, in vitro reagent, or other similar or related article, including a component part, or accessory which is: (1) recognized in the official National Formulary, or the United States Pharmacopoeia, or any supplement to them; (2) intended for use in the diagnosis of disease or other conditions, or in the cure, mitigation, treatment, or prevention of disease, in man or other animals, or intended to affect the structure or any function of the body of man or other animals; and (3) which does not achieve any of its primary intended purposes through chemical action within or on the body of man or other animals and which is not dependent upon being metabolized for the achievement of any of its primary intended purposes. ("What is a Medical Device?," 2015)

This study deals with medical devices that are based on IoT technologies. It focuses on the attitudes of physicians toward adoption of IoT medical devices (IoT-MDs) and the impacts these devices have on medical practice during patient visits. 


\section{Theoretical Background}

According to the Technology Acceptance Model (TAM), psychological factors that impact acceptance of new technologies can be measured. For this purpose, Venkatesh, Morris, Davis, and Davis (2003) proposed the Unified Theory of Acceptance Use of Technology (UTAUT) model, in which gender, age, experience and voluntariness to use, are the four primary factors. Research regarding age differences in individual decisions in China shows that young people tend to adopt new technology faster than old people do ( $\mathrm{Lu}, \mathrm{Yu}, \& \mathrm{Liu}, 2006)$. US researchers found that for older people, a crucial factor in using technology is the interface - easy reach, maximal transparency, and minimal ambiguity are paramount. Eventually however, older people will want to learn the new technology ( $\mathrm{Lu}$ et al., 2006). It can be said that an individual's willingness to adopt a new technology will be affected by the intensity of exposure, which itself influences other factors, such as the level of clarity felt toward the technology (Khalifa \& Cheng, 2002; Picoto, Bélanger, \& Palma-dos-Reis, 2014). In addition to the human factors, methods of application should also be considered.

In any instance of adopting new technologies for monitoring patient status at a distance, the most important factor appears to be the existence of supportive technical infrastructures. This is exemplified in the decision of healthcare professionals to use teledermatology, a subspecialty of dermatology, and one of the most common applications of telemedicine and e-health (Gagnon, Orruño, Asua, Abdeljelil, \& Emparanza, 2012).

Thanks to advances in sensory technologies, many bio-medical sensors can measure certain clinical parameters of patients effectively, store them, and make them available for access anytime, anywhere (Sankar Bhunia, 2015). Notable among such devices are: "Gluco phones", a device for chronic diabetic patients, which are capable of transferring real-time glucose patient status to the appropriate health authorities (West, 2012); "UroSense," a medical device system that automates urine output as well as core body temperature data gathering and charting for catheterized patients (Link Labs, 2015). Another example of an IoT medical product is "Embrace", which measures stress, epileptic seizures, activity and sleep (Bidwell, Khuwatsamrit, Askew, Ehrenberg, \& Helmers, 2015). As the list grows, so does the debate surrounding this subject.

Predictably, advances in technological systems have produced conflicts of opinions among doctors. On one hand, research into information and communication technologies shows doctors to be supportive of the IoT trend. Some doctors stated that they believe patients will increasingly favor IoT devices, and some presented the benefits of using this technology, noting primarily the increase in doctor-patient efficiency, which will percolate to affect the community as a whole (Hanna \& Fairhurst, 2013). The time needed to take primary physical measurements is only $8 \%$ of visiting length, however these durations aggregate to several monthly hours per physician. Some IoT-MDs can reduce visitation length, as they can take medical readings beforehand (Rhodes, McFarland, Finch, \& Johnson, 2001). On the other hand, a growing group of doctors is questioning the potential of the technology to influence treatment effectively. They argue that increased integration of IoT devices will negatively affect social aspects of medical practice (Hanna \& Fairhurst, 2013). 
One factor contributing to both sides of the debate is the growing use of social networks, which allows patients to share information, experience, etc. (Boyd, 2011; Goldberg, 2010; Mohammed, 2012). This phenomenon, however, does not necessarily reduce the doctor's relevance. Although the patient is more independent regarding his own health, the doctor is not perceived as obsolete (Christmann, 2013). Adding to this independence are IoT devices that can notify hospitals of patient status, thus relieving patients from routine doctor visits. Similar devices can also remind patients when to take their medication and at what quantities (Santucci, 2010). However, the increasing availability of medical knowledge appears to have negative consequences as well, with some doctor testifying that they have felt offended in situations where they were forced to confront informed patients. They also report longer consultation and session times, and consequently greater effort being spent on explaining medical jargon to exceedingly worrying patients (Christmann, 2013). Moreover, the healthcare industry, in contrast to other industries, is the only one to resist the use of widespread online communication technologies (Dixon, 2010). Overall, it can be said that new technology does influence doctor-patient relationships, but not to a degree where the balance of power is greatly shifted (Christmann, 2013). However, this may change in the future, as the IoT field rapidly permeates society.

Growth in smartphone availability has opened possibilities for their use in healthcare in conjunction with IoT devices. For instance, applications intended to evaluate a patient's status, and suggest treatments based on long-term observations and professional council, are becoming abundant (Luxton, McCann, Bush, Mishkind, \& Reger, 2011). The IoT can facilitate additional, non-traditional medical environments, such as portable clinics and home care services (Luxton et al., 2011). These, as well as the growing trend of telehealth, make use of telemonitoring, which consists of remote consultations with physicians over mobile devices or desktop computers (Terry, 2015). These are primarily employed for follow-ups, medication consultation, and ongoing treatments for patients with chronic medical conditions. Physicians recognize the potential of these systems for saving time and improving the quality of life of both patients and physicians. Some physicians are even involved in telehealth initiatives because they believe that virtual meetings have the potential to be as effective as face-to-face meetings (Terry, 2015). Virtual meetings can also reduce costs for patients, physicians and for the entire health care system (Dixon \& Rao, 2014).

Based on market trends, the application of medical technologies is expected to grow in the future. Our society is evolving, becoming more mobile and developing an increased awareness of health. The growth in smartphone use could make healthcare more accessible, efficient and interactive for controlling and connecting to IoT-MDs (Luxton et al., 2011). There are numerous examples of budding IoT-based medical solutions and devices, with many emerging daily, and what will happen in the near future is abundantly clear - more and more actions will become automated. IoT devices will make medical systems better and more efficient in the foreseeable future (Halliday \& Hohberger, 2012). However, the true vision of the IoT phenomenon speaks of a mélange of small applications converging into a greater whole:

Imagine if you are a relative of a patient who forgot their medicine. You receive the alert, are able to know their location, check their vital signs remotely to see if they are falling ill, then be informed by your car's navigation system which hospital has the most free beds, the clearest traffic route to get there and even where you can park. (Niewolny, 2013, p. 6) 


\section{Research Methodology}

The subject of this research is new and, insofar as the authors are aware, has not yet been explored; therefore, an exploratory study was required. Mixed-methods research is a useful approach in such cases in order to better clarify and explain the field of research, as well to discover new perspectives on it (Greene, Caracelli, \& Graham, 1989). The goal of this study was to shed light on the following major questions:

RQ1. What are the attitudes of physicians toward IoT-MDs in their practices?

RQ2. Is there a difference in attitudes between physicians who have been exposed to IoT-MDs and those who have not?

RQ3. Is there any significant change in attitudes of physicians between 2014 and 2015 ?

The study was conducted in seven phases:

The authors could not find an existing questionnaire that addresses all aspects of this research, therefore, in accordance with the exploratory nature of the study, a questionnaire was independently constructed, with partial reliance on the UTAUT model (Venkatesh et al., 2003). This pilot questionnaire was distributed to 23 physicians, who agreed not only to answer the questions, but also to address the quality of the questionnaire - the relevance of the questions, their clarity, and any other aspect respondents wished to comment on. Based on the pilot results and remarks of the participants, the questionnaire was modified to better adapt it to the survey's population while also increasing its validity and reliability. During December 2014 the modified questionnaire was distributed to 126 physicians via social networks, professional forums on the Internet, medical centers, hospitals, and personal acquaintances. A diverse group of physicians, all distinct from the 23 who participated in the pilot, responded to the modified questionnaire. In parallel, personal interviews were conducted with four physicians varying in field of expertise, seniority, and age. It was made clear to all respondents that this research deals exclusively with FDA approved IoT-MDs. Responses to the modified questionnaire and interviews were analysed and some conclusions were drawn. About a year later, during December 2015, another 50 physicians from the same kinds of organizations as those surveyed in 2014, responded to the same modified questionnaire. Finally, the new data was analysed and compared to the previous responses. Conclusions were drawn based on the combined survey results and the comparison between the two surveys.

Two data-collection tools were employed in this study: a questionnaire, and personal, semistructured interviews. The interviews were conducted face-to-face and were simultaneously transcribed. Each interview consisted of open-ended questions, which allowed interviewees to present their views on the subject in both breadth and depth. Interviews examined, among other things, the extent to which the physician and his/her colleagues were acquainted with IoT-MDs, the specific ways physicians use such devices, the impact of the devices on their medical practice during patient visits, barriers to using IoT-MDs such as physician concerns, and forecasts for the use and impact of this technology in the future.

The questionnaire was constructed while considering relevant aspects of the UTAUT model, including the use of moderating variables such as age, gender and intention to use (Venkatesh et al., 2003). The questions were adapted to our study, for example, by taking into account that the subject of our research is a family of devices, and not a single, specific system. 
The questionnaire contained two question types: structured questions intended to provide measurable data for quantitative analysis, and open-ended questions intended to provide broader information, which was desirable due to the exploratory nature of this pilot research project. The questionnaire also consisted of two main sections. The first section included six items referring to demographic and basic data. The second section addressed the physician's attitudes and opinions, and was aimed at answering RQ1 (the question regarding physicians' attitudes toward IoT-MDs in their practice), by focusing on the following secondary questions:

1. Are physicians aware of various IoT-MDs? Do they find them reliable? Does using IoTMDs by physicians result in shorter patient visits?

2. Are physicians concerned about treating and dealing with patients who are, in their opinion, well informed about their medical condition?

3. Does the patient's level of understanding impact the length of the visit?

4. Is there a correlation between the physician's age and his/her willingness to engage with IoT-MDs?

In order to answer all four secondary questions, the second section of the questionnaire consists of items referring to these areas: current medical practice, attitudes toward patients, attitudes toward IoT-MDs, and attitudes toward IoT-MDs as part of medical practice.

\section{Sampling}

Convenience (or opportunity) sampling is a convenient way to collect data on a limited financial budget (Sanders \& Liptrot, 1993). The current research was not only restricted in budget, but medical organizations, whose cooperation was needed in order to achieve a probability sample, imposed bureaucratic demands. They insisted that the researchers provide the same authorizations used for medical research projects, despite the fact that the requested responses were unrelated to patient medical information. Therefore, physicians were instead contacted directly, though this resulted in nonprobability sampling, which prevents the generalization of results, but is often used in exploratory research projects. The sampling of the physician population took into consideration the need to include a wide variety of parameters that could potentially impact decision making by physicians and their attitudes toward technology and patient care. Such parameters include: gender, age, seniority, area of specialization, as well as the type and identity of organizational affiliation.

\section{Data Collection}

Questionnaire data was collected through Google Forms. The physicians who agreed to participate in this study provided their responses in one of three ways: directly online, on a printed hard-copy version, or over the telephone. $21.6 \%$ of the responses were filtered out, either because of the respondent being unsuitable to the target population, or due to internal inconsistencies - providing logically conflicting responses to related questions. After filtering, 138 responses remained in total, of which 95 were from the survey of 2014, and 43 from 2015. The demographic characteristics of participants in each survey differed statistically to a small degree as can be observed in Table 1 . 
As mentioned above, this research did not involve patients nor was any personal health information collected. Participating physicians were assured response anonymity and no personal identification data was collected. Consequently, there were no ethical constraints on this research, and no approval was needed from ethics regulatory committees.

Table 1. Demographic Analysis

\begin{tabular}{|c|c|c|c|c|c|c|}
\hline \multirow[t]{2}{*}{ Characteristic } & \multicolumn{2}{|c|}{ 2014-2015 } & \multicolumn{2}{|c|}{2014} & \multicolumn{2}{|c|}{2015} \\
\hline & $\mathbf{n}$ & $\begin{array}{c}\begin{array}{c}\text { Percent of } \\
2014-2015 \\
(n=138)\end{array}\end{array}$ & $\mathbf{n}$ & $\begin{array}{c}\text { Percent of } \\
2014 \\
(n=95)\end{array}$ & $\mathbf{n}$ & $\begin{array}{c}\text { Percent of } \\
2015(n=43)\end{array}$ \\
\hline 1. Gender & & & & & & \\
\hline a. $\quad$ Male & 67 & $48.60 \%$ & 48 & $50.50 \%$ & 19 & $44.20 \%$ \\
\hline b. Female & 71 & $51.40 \%$ & 47 & $49.50 \%$ & 24 & $55.80 \%$ \\
\hline 2. Age & & & & & & \\
\hline a. $\quad 25-32$ & 29 & $21.01 \%$ & 20 & $21.10 \%$ & 9 & $20.93 \%$ \\
\hline b. $\quad 33-40$ & 26 & $18.84 \%$ & 19 & $20.00 \%$ & 7 & $16.28 \%$ \\
\hline c. $41-48$ & 26 & $18.84 \%$ & 19 & $20.00 \%$ & 7 & $16.28 \%$ \\
\hline d. $\quad 49-56$ & 40 & $28.99 \%$ & 28 & $29.50 \%$ & 12 & $27.91 \%$ \\
\hline e. $\quad 57$ and above & 17 & $12.32 \%$ & 9 & $9.50 \%$ & 8 & $18.60 \%$ \\
\hline 3. Seniority & & & & & & \\
\hline a. less than 5 years & 40 & $29.00 \%$ & 29 & $30.50 \%$ & 11 & $25.60 \%$ \\
\hline $\begin{array}{ll}\text { b. } & 5-9\end{array}$ & 9 & $6.50 \%$ & 6 & $6.30 \%$ & 3 & $7.00 \%$ \\
\hline $10-14$ & 14 & $10.10 \%$ & 11 & $11.60 \%$ & 3 & $7.00 \%$ \\
\hline $\begin{array}{ll}\text { d. } & 15-19\end{array}$ & 18 & $13.00 \%$ & 16 & $16.80 \%$ & 2 & $4.70 \%$ \\
\hline e. $20-24$ & 29 & $21.00 \%$ & 22 & $23.20 \%$ & 7 & $16.30 \%$ \\
\hline f. more than 24 years & 28 & $20.30 \%$ & 11 & $11.60 \%$ & 17 & $39.50 \%$ \\
\hline 4. Type of organization & & & & & & \\
\hline $\begin{array}{l}\text { a. Institution } \\
\text { (hospital/army/nursing home) }\end{array}$ & 58 & $42.00 \%$ & 44 & $46.30 \%$ & 14 & $32.60 \%$ \\
\hline b. $\mathrm{HMO}$ & 76 & $55.10 \%$ & 53 & $55.80 \%$ & 23 & $53.50 \%$ \\
\hline c. $\quad$ Private clinic & 25 & $18.10 \%$ & 17 & $17.90 \%$ & 8 & $18.60 \%$ \\
\hline
\end{tabular}




\section{Analysis}

The data collected in the survey and in the interviews was analyzed using a mixed-methods technique. Open-ended questions, both from the interviews and from the questionnaire were subjected to content analysis, while structured questions were analyzed using quantitative methods. Content analysis was intended to shed light on the open questions, both from the interviews and the surveys, in order to provide information about such aspects as the attitudes of participants toward IoT-MDs and their impacts, as well as the attitudes toward informed patients. Quantitative analysis was done by using both pivot tables in Microsoft Excel, and statistical calculations in SPSS exclusively on answers to structured questions of the questionnaire. In order to answer research questions, RQ2 and RQ3, these statistical analysis techniques were used for each one of the surveys separately and also on the two surveys combined, for comparison purposes.

\section{Results}

Quotations in this section derive from content analysis of both interviews and of open questions in the questionnaire. They are presented in order to provide a better understanding of various aspects of RQ1 - attitudes of physicians toward IoT-MDs in their practice.

\section{Attitudes Toward IoT-MD}

Concerning the reliability of IoT-MDs, $49.3 \%$ of the respondents find such devices reliable, $12.3 \%$ view them as unreliable, while $38.4 \%$ are not sure. One of those interviewed explained that an FDA approval of a broad variety of products would change doctors' willingness to count on varied devices used by patients, while adding that processes in the medical field are very prolonged and can take many years. $67.4 \%$ of respondents believe that IoT-MDs can substitute for existing medical products, but of these, only $68.8 \%$ would recommend that their patients obtain such devices.

Some of the respondents referred to the possibility that IoT-MDs would bring about a revolution in healthcare in the future. Their statements can be subdivided into several categories. The first includes general comments, such as: "Any device that is strongly deployed could have a strong revolutionary impact on the field of medicine," or "It will bring medicine to a new and better place." Another category of comments refers to the revolutionizing impact of enhanced doctorpatient relationships, for example: "The products will create a new form of communication with patients, allowing communication as equals," or "It will allow an increase in remote treatments." In a third category, revolutionary impacts were associated with the implications on quality of care: "If a patient can transmit measurements remotely, it allows substantive and rapid response by the physician. It is substantially quicker, which is critical in some pathological situations," and "In the world of surgery, some (IoT) surgical instruments have the potential to help us discover problems faster and resolve them more quickly not only during patient visits, but during surgery as well. After surgery, follow-up would also be more accurate."

Other participants took a more critical approach. Their statements can also be subdivided into multiple categories. With regard to information the patient receives, comments include: "Providing continuous information to the patient about his blood pressure level could cause him 
stress and interfere with his daily life. There is no need for continuous information about everything. Even for diabetes - it is preferable to provide patients only with exceptional measurements rather than continuous information in order not to disturb their daily routine. One needs to remember that the purpose of monitoring and treatment is to allow the patient to lead a normal life and to cause minimal disruption." In reference to the doctor-patient relationship, critical comments include: "There is no substitute for a real doctor-patient connection, so that products can aid but not replace it." Another physician referred to the quantity of testing: "It would lead to unnecessary testing due to an overly high level of awareness that is not always required." Other participants simply do not consider the adoption of IoT-MDs revolutionary: "I think it can upgrade medical monitoring in certain situations and for certain patients only" or "not particularly revolutionary...just another tool in the toolbox."

Some of the respondents expressed concerns about IoT-MDs, and others made qualified statements regarding the revolutionary nature of such products and possible barriers to their widespread adoption: "It depends on whether physicians will trust these products," or: "Right now, unfortunately there is still no awareness among doctors... due to both technological development and because regulation has not changed attitudes....I hope that no dangerous mistakes will be made," and finally, "We must not fear technology, but should embrace it and see how it can streamline our workflow and patient care."

\section{Medical Practice in General and in Conjunction With IoT-MDs}

Time spent taking patient measurements may impact physicians' attitudes toward deployment of IoT-MDs. $83.3 \%$ of the respondents claim that taking measurements prolongs visits. $94.8 \%$ of these physicians believe that IoT-MDs can shorten patients' visits. Yet, $16.5 \%$ of the latter state that they would always take their own measurements, even if the patient were using an IoT-MD. In one interview, a family physician explained that taking continuous measurements in the patient's natural environment produces more accurate results and neutralizes the "white coat hypertension" phenomenon.

There is a very broad consensus among the survey's participants that in the near future the continuing research and development of IoT-MDs will streamline the workflow of patient visits. The main expected contribution of IoT-MDs to the practice of medicine according to $42.8 \%$ of participants would be the early warning that both patient and doctor would receive in the case of exceptional measurements. Additional key benefits would include reduction in the number of visits aimed at routine examinations $(37.7 \%)$, and a new form of doctor-patient communication that would be based on real-time data (31.2\%). Several respondents also suggested a number of other lesser benefits.

Most of the physicians who claimed that they would always take manual measurements of their own indicated that they would change this habit if the use of IoT-MDs were to become part of their organizations' regulations $(29.5 \%)$, or if they were more knowledgeable about research and development of such devices $(28.4 \%)$.

$62 \%$ of the participants declared that they were in favor of integrating IoT-MDs in healthcare while only $4.7 \%$ were against. However, only $39.9 \%$ of the respondents supported the use of IoT-MDs in medical practice. These participants not only expressed willingness to integrate the devices into their practice, but they did not question the reliability of IoT-MDs as well as 
claimed that they could shorten patients' visits and increase the physician's efficiency. On the opposing side, $38.4 \%$ of respondents showed a high level of concern about integrating IoT-MDs in their practice. These physicians do not believe that currently available IoT-MDs are reliable enough, and they prefer to take their own measurements during patient's visits without counting on IoT-MDs regardless of whether or not the patient uses such a device.

\section{Attitudes Toward Informed Patients}

Examining physicians' attitudes toward patients who are informed by IoT-MD outputs, shows that $72.5 \%$ claimed they either had no objection or were slightly reluctant to treat such patients. To other aspects, a vast majority of the participants responded positively. $81.9 \%$ of all respondents believe that a patient who has an IoT-MD could contribute to treatment. On the other hand, $13.8 \%$ say that such patients may impair the treatment process, $6.5 \%$ see such patients who hold very precise data, as paranoid, and a few even claim that the ideal patient is one who has no preliminary knowledge (3.6\%). One of those interviewed described some patients "as if they were coming to an auto mechanic asking for a specific treatment."

Doctors are at odds over the impact of IoT-MDs on the nature of a patient's visit. Some point out that it may prolong visits because of multitudes of patient questions and demands for more indepth explanations (31.9\%). Others think visits would be shorter, either because they would become more pragmatic $(29.7 \%)$, or because they would shorten explanations of test results.

\section{Exposure to IoT-MDs}

$62.3 \%$ of the participants had been exposed to IoT-MDs prior to the survey. A comparison between participants who had been exposed to IoT-MDs and those who had not, shows a few non-significant differences with regard to demographic characteristics. For instance, average seniority: 14.46 years for the exposed group, and 16.25 years for the unexposed, or gender: $62.7 \%$ of the males, and $62 \%$ of the females were exposed. However, there are some significant differences between the two groups. As can be seen in Figure 1, physicians who work for HMOs and in private clinics are more exposed than those who are employed by institutions such as hospitals and the military. There is also an age difference: in all age groups there was a majority of doctors who were exposed to IoT-MDs, however older physicians (aged 41+) were exposed more than younger ones (aged 25-40). These findings are illustrated in Figure 2.

Physicians with prior exposure expressed greater willingness to incorporate this technology into their medical practice. These findings are based on the sum of scores participants gave to four statements concerning various aspects of willingness to adopt IoT technology, with scores of 0 (against) through 8 (in favor). This phenomenon is statistically significant $(p<0.005)$, and it is revealed in Figure 3. Another finding suggests that physicians with prior exposure are more likely to recommend that patients obtain IoT-MDs (60.5\% versus $23.1 \%)$. 


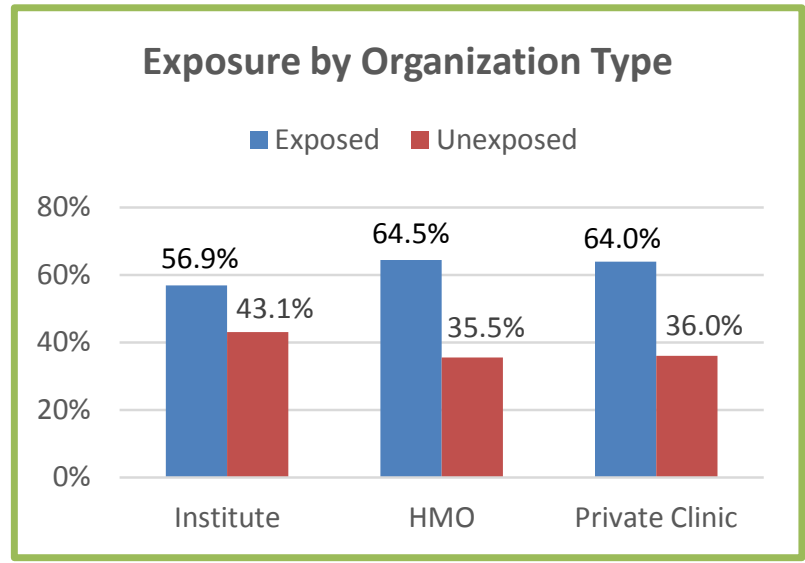

Figure 1. Exposure by Organization Type

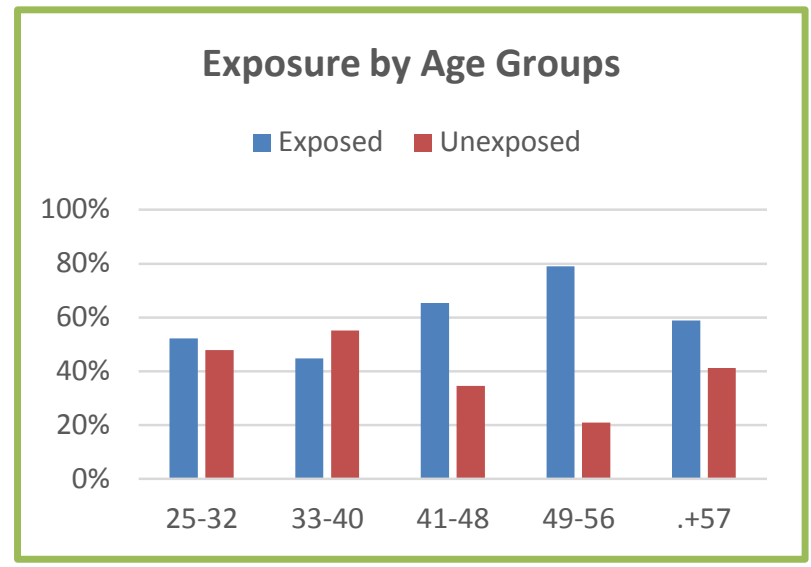

Figure 2. Exposure by Age Groups

\section{The Age Factor}

There was a significant negative correlation $(r s=-0.216, p<0.05)$ between age and willingness to incorporate IoT-MDs into medical practice. Figure 4 depicts these results: participants are grouped by age, and willingness is scored from 0 (against) to 8 (in favor).

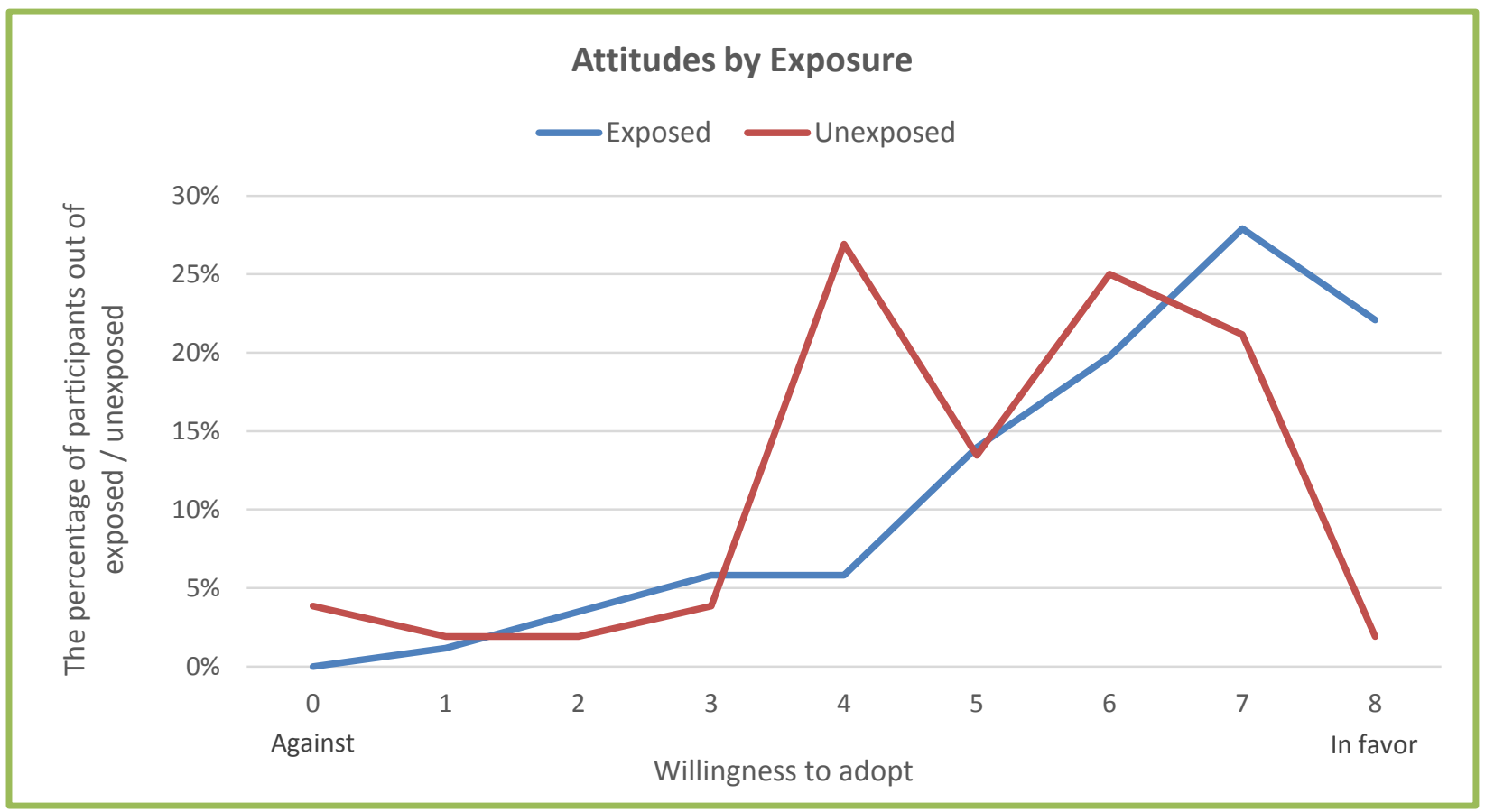

Figure 3. Attitudes by Exposure 


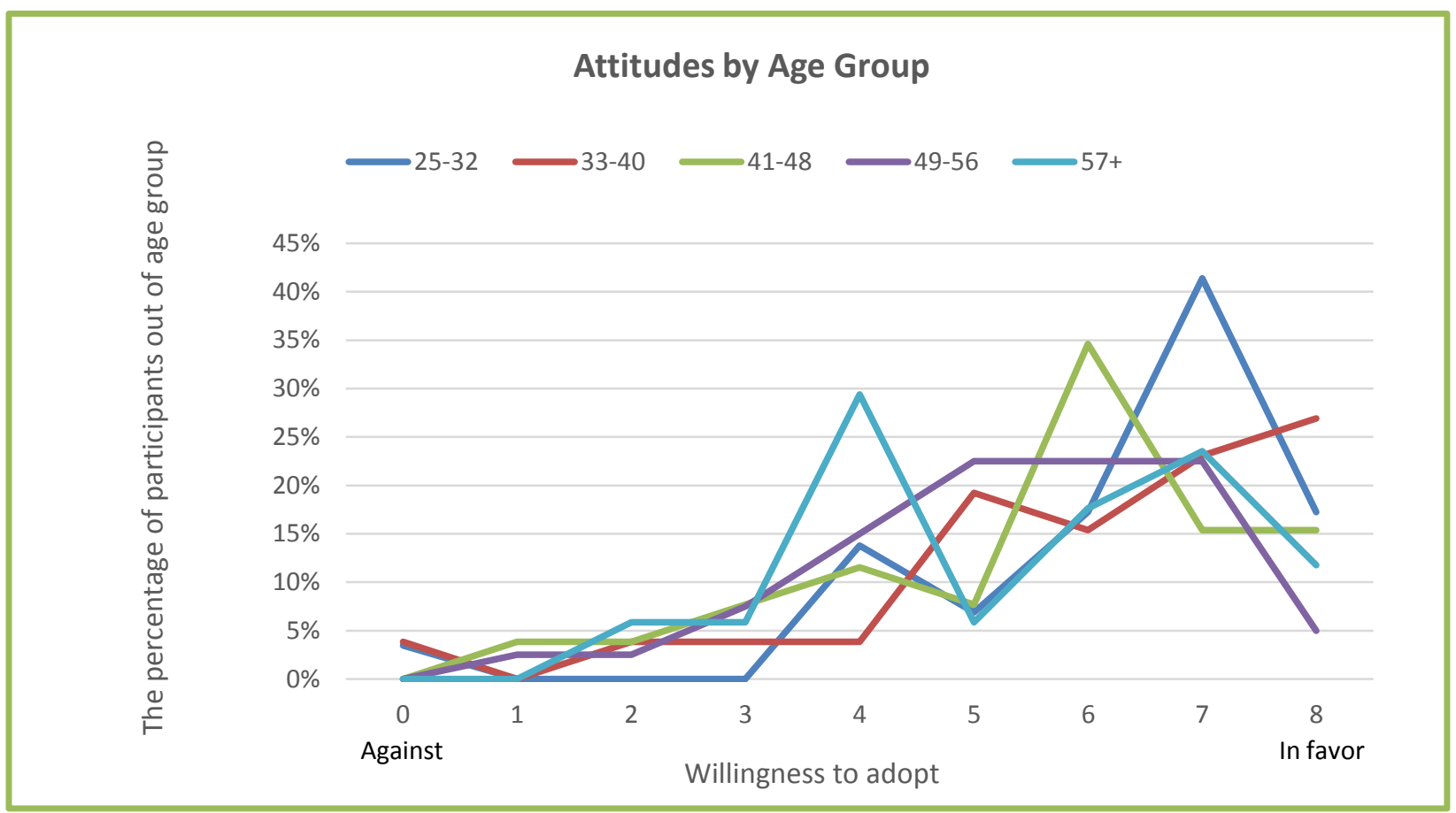

Figure 4. Attitudes by Age Group

\section{A Comparison Between Attitudes of Physicians in 2014 and 2015}

As mentioned above, two surveys were taken, one in December 2014 and the other, a year later. The same questionnaire was distributed, and results show some differences in responses. The most significant differences are summarized in Table 2. As can be observed, the participants of the later survey have more experience in their profession, a smaller percent of them work in hospitals and similar institutions, and a much smaller percent of them had prior exposure to IoTMDs. Respondents in 2015 showed negative attitudes toward IoT-MDs more than in 2014. This is revealed from the following survey items: A lower percent of doctors who would recommend that their patients obtain IoT-MDs. More doctors do not think that IoT-MDs can shorten visits, nor that they will replace other medical products currently in use. Fewer doctors would skip manually measuring patients with IoT-MDs.

Another interesting difference between the two surveys appears in statistical correlations. Specifically, in the 2015 survey, no correlation was found between the age of participants and their attitude as calculated based on the four statements mentioned above, nor between the sum of the four statements and having had prior exposure to IoT-MDs.

On the other hand, in the 2014 survey a significant negative correlation $(r s=-0.294, p<0.01)$ was found between age and attitude, and a correlation was found between the sum of the four statements and prior exposure $(p<0.05)$. 
Table 2. Differences between the Surveys

\begin{tabular}{|c|c|c|}
\hline Survey Item & 2014 responses & 2015 responses \\
\hline Seniority & 13.4 years & 18 years \\
\hline $\begin{array}{l}\text { Employee of an institution (hospital/army/ } \\
\text { nursing home) }\end{array}$ & $46.3 \%$ & $32.6 \%$ \\
\hline Prior exposure to IoT-MDs & $70.5 \%$ & $44.2 \%$ \\
\hline $\begin{array}{l}\text { IoT-MDs will not replace current medical } \\
\text { products }\end{array}$ & $20.0 \%$ & $30.2 \%$ \\
\hline IoT-MDs cannot shorten routine visits & $9.5 \%$ & $14.0 \%$ \\
\hline $\begin{array}{l}\text { Will not take manual measurements from a } \\
\text { patient with an IoT-MD }\end{array}$ & $38.9 \%$ & $30.2 \%$ \\
\hline Recommend that patients obtain IoT-MDs & $49.5 \%$ & $39.5 \%$ \\
\hline
\end{tabular}

\section{Discussion and Conclusions}

This research examines attitudes of physicians toward medical IoT-MDs and their integration into medical practice. Combining information from our two surveys allows identifying the primary differences between physicians with positive versus negative attitudes toward IoT-MDs and their use. The existence of different positions on this issue is not surprising and fits Hanna and Fairhurst's (2013) findings. Some of the participants of our research declared that they were in favor but actually expressed opposition to the products and their integration. Physicians with the most positive attitudes in our research believe in the potential of IoT technology and foresee that such devices will affect medical practice by shortening and optimizing patient care. They trust the reliability of the devices and would recommend that their patients obtain them. This trend, expressed by $21 \%$ of participants, is significantly stronger among females (55.2\%), younger doctors (average age of 44.1 years) with less seniority (13.3 years), than the doctors against. Physicians with a very negative attitude toward IoT-MDs would perform examinations without relying on the devices' results. They are against integration of the IoT-MDs into their practice and would not recommend that their patients obtain them. This group, which contains $18.8 \%$ of participants, is characterized by more males $(53.8 \%)$ who are older (average age of 48.3 years) with higher seniority (18.2 years).

The difference in openness to adapting technology in correlation to age, as presented here, fits Venkatesh et al. (2003). In contrast, the corresponding correlation found with gender, where females are more open with respect to technology than males, does not fit Wilkowska, Gaul, \& Ziefle (2010) who found that there was no significant difference in users' willingness to use medical technology based on gender.

The most significant correlation found relates to doctors' prior exposure to IoT-MDs. Of those with an extremely positive attitude, $90 \%$ had been previously exposed to this technology, while only $46 \%$ of those with extremely negative attitudes had been exposed. These findings fit with Khalifa and Cheng (2002), as well as Picoto et al. (2014). 
Comparing the primary differences between the surveys of 2014 and 2015 reinforces the hypothesis that exposure has very significant influence on all other variables. There is no evidence in our data that the time lapse between the two surveys, on its own, resulted in increased awareness or openness of physicians to IoT technology. This would imply that, in order to generate positive attitudes toward IoT technology in healthcare and its integration into practice, doctors' exposure to it should be increased. In addition, making physicians more knowledgeable about research and development of IoT-MDs would be expected to increase their perception of the reliability of such products.

Reactions received from many of the participants are consistent with findings that increased use of IoT-MDs enhance patient-doctor collaboration (Terry, 2015), reduce costs (Dixon \& Rao, 2014; Terry, 2015) and make medical systems more efficient (Halliday \& Hohberger, 2012). With the fast growing trend of telemonitoring, one can expect the deployment of IoT-MDs in healthcare to become more significant in the near future.

The authors believe that data generated from IoT-MDs is currently too raw for practical use. Developing supportive systems with a friendly user experience and APIs to the most popular IoT-MDs might help physicians overcome the technological barrier caused by the lack of integrated tools (Dixon, 2010). Such systems should extract and highlight measured irregularities in order to increase physicians' openness to IoT-MDs, and to enable medical practice to be more efficient. They should produce comprehensive, easy to understand charts of the patient's health condition, with capabilities to zoom-in for detailed results, and zoom-out for a broader picture. Such systems are expected to increase both product effectiveness and physician efficiency affecting the whole field of healthcare, and telemedicine in particular.

\section{Limitations and Future Research}

The authors recommend further research into both awareness and attitudes of physicians as IoTMDs improve and become more widespread. Extending research into IoT-MD challenges, security, privacy, regulatory barriers worldwide, handling of massive data, etc., may also contribute to a better understanding of the results. The current research took place in Israel, and used a nonprobability sampling. In order to increase generalization of the findings and improve their quality, studies using complete random sampling as well as international data collection are recommended. Another potentially interesting direction is to research the mutual influences of IoT-MD use and the development and implementation of information systems aimed at presenting aggregated data collected by these products.

\section{Acknowledgments}

The authors would like to thank all the physicians who took part in this research, filled in the questionnaires, were interviewed and allowed us to gain insights into this field. 


\section{References}

Ashton, K. (2009). That 'Internet of things' thing. RFiD Journal. Retrieved December 2015, from http://www.rfidjournal.com/articles/view?4986

Bidwell, J., Khuwatsamrit, T., Askew, B., Ehrenberg, J. A., \& Helmers, S. (2015). Seizure reporting technologies for epilepsy treatment: A review of clinical information needs and supporting technologies. Seizure: European Journal of Epilepsy, 32, 109-117.

Boyd, D. M. (2011). Social Sites as Networked Publics: Affordances, Dynamics, and Implications. In Z. Papacharissi (Ed.), Networked Self: Identity, Community, and Culture on Social Network Sites (pp.39-58). New York. Routledge.

Christmann, S. (2013). The impact of online health information on the doctor-patient relationship. (Master's Thesis). Retrieved from Media@lse Electronic MSc Dissertation Series.

Couturier, J., Sola, D., Scarso Borioli, G., \& Raiciu, C. (2012). How can the Internet of things help to overcome current healthcare challenges. Communications and Strategies, 87, 67-81.

Dixon, R. F. (2010). Enhancing primary care through online communication. Health Affairs, 29(7), 1364-1369.

Dixon, R. F., \& Rao, L. (2014). Asynchronous virtual visits for the follow-up of chronic conditions. Telemedicine and e-Health, 20(7), 669-672.

Fundación de la Innovación Bankinter. (2011). The Internet of Things in a connected world of smart objects. Retrieved January 2016, from https://www.fundacionbankinter.org/documents/11036/16211/Publicacion+PDF+IN+ FTF_IOT/75dde4a5-183e-4f1c-b7cd-ab0cdf4b738a

Gagnon, M. P., Orruño, E., Asua, J., Abdeljelil, A. B., \& Emparanza, J. (2012). Using a modified technology acceptance model to evaluate healthcare professionals' adoption of a new telemonitoring system. Telemedicine and e-Health, 18(1), 54-59.

Gantz, J., \& Reinsel, D. (2012). The Digital Universe In 2020: Big Data, Bigger Digital Shadows, and Biggest Growth in the Far East. Retrieved January 2016, from http://www.emc.com/collateral/analyst-reports/idc-the-digital-universe-in-2020.pdf

Goldberg, R. (2010). Tabloid medicine: how the Internet is being used to hijack medical science for fear and profit. New-York: Kaplan Publishing. 
Greene, J. C., Caracelli, V. J., \& Graham, W. F. (1989). Toward a conceptual framework for mixed-method evaluation designs. Educational Evaluation and Policy Analysis, 11, $255-274$.

Gubbi, J., Buyya, R., Marusic, S., \& Palaniswami, M. (2013). Internet of things (IoT): A vision, architectural elements, and future directions. Future Generation Computer Systems, 29(7), 1645-1660.

Halliday, S., \& Hohberger, C. (2012). Healthcare and the impact of the Internet of things. Association for Automatic Identification and Mobility. Retrieved January 2016, from http://c.ymcdn.com/sites/www.aimglobal.org/resource/resmgr/docs/aim_iot_healthcar e_whitepape.pdf

Hanna, L., \& Fairhurst, K. (2013). Using information and communication technologies to consult with patients in Victorian primary care: The views of general practitioners. Australian Journal of Primary Health, 19(2), 166-170.

Khalifa, M., \& Cheng, S. (2002). Adoption of mobile commerce: role of exposure. Proceedings of the $35^{\text {th }}$ Annual Hawaii International Conference on System Sciences 2002.

Laranjo, I., Macedo, J., \& Santos, A. (2012). Internet of things for medication control: Service implementation and testing. Procedia Technology, 5, 777-786.

Link Labs. (2015, January). 3 IoT healthcare apps. Retrieved January 2016, from http://www.link-labs.com/iot-healthcare/

Lu, J., Yu, C. S., \& Liu, C. (2006). Gender and age differences in individual decisions about wireless mobile data services: A report from China. University of Houston-Victoria. Retrieved December 2015, from http://helsinkimobility.aalto.fi/papers/Mobile\%20Services_1_3.pdf

Luxton, D. D., McCann, R. A., Bush, N. E., Mishkind, M. C., \& Reger, G. M. (2011). mHealth for mental health: Integrating smartphone technology in behavioral healthcare. Professional Psychology: Research and Practice, 42(6), 505-512.

Mohammed, S. N. (2012). The (dis) information age: The persistence of ignorance. New York: Peter Lang Publishing.

Niewolny, D. (2013). How the internet of things is revolutionizing healthcare. Retrieved January 2016, from https://cache.freescale.com/files/corporate/doc/white_paper/IOTREVHEALCARWP.p df 
Picoto, W. N., Bélanger, F., \& Palma-dos-Reis, A. (2014). An organizational perspective on m-business: Usage factors and value determination. European Journal of Information Systems, 23(5), 571-592.

Phan, T. A. M. (2013). Cloud Databases for Internet-of-Things Data. (Master's thesis, Technical university of Denemark).

Rhodes, D. R., McFarland, K. F., Finch, W. H., \& Johnson, A. O. (2001). Speaking and interruptions during primary care office visits. Family Medicine-Kansas City, 33(7), 528-532.

Sanders, P., \& Liptrot, D. (1993). Incomplete Guide to Basic Research Methods and Data Collection for Counsellors. Manchester: PCCS.

Sankar Bhunia, S. (2015). Adopting Internet of things for provisioning health-care. Proceedings of the 2015 ACM International Joint Conference on Pervasive and Ubiquitous Computing and Proceedings of the 2015 ACM International Symposium on Wearable Computers (pp. 533-538). ACM.

Santucci, G. (2010). The Internet of Things: Between the Revolution of the Internet and the Metamorphosis of Objects. In H. Sundmaeker, P. Guillemin, P. Friess, \& S. Woelfflé (Eds.), Vision and challenges for realising the Internet of things (pp. 11-24). Belgium: CERP - IoT.

Terry, K. (2015). Physicians and telehealth: Is it time to embrace virtual visits? Medical Economics, 92(13), 48.

Top 10 communications technology trends in 2015. (2015). IEEE Communications Society. Retrieved December 2015, from http://www.comsoc.org/blog/top-10communications-technology-trends-2015

Venkatesh, V., Morris, M. G., Davis, G. B., \& Davis, F. D. (2003). User acceptance of information technology: Toward a unified view. MIS Quarterly, 27(3), 425-478.

West, D. (2012). How mobile devices are transforming healthcare. Issues in Technology Innovation, 18, 1-14.

What is a medical device? (2015). U.S. Food and Drug Administration. Retrieved April 2016, from http://www.fda.gov/AboutFDA/Transparency/Basics/ucm211822.htm

Whitmore, A., Agarwal, A., \& Da Xu, L. (2015). The Internet of things - A survey of topics and trends. Information Systems Frontiers, 17(2), 261-274. 
Wilkowska, W., Gaul, S., \& Ziefle, M. (2010). A small but significant difference - The role of gender on acceptance of medical assistive technologies. In G. Leitner, M. Hitz, \& A. Holzinger (Eds.), HCI in Work and Learning, Life and Leisure (pp. 82-100). Springer Berlin Heidelberg.

\section{Authors' Biographies}

Dafni Biran Achituv teaches in the Information Systems B.Sc. program at The Academic College of Tel Aviv Yaffo. She holds an M.A. in Organizational Consulting and Development (summa cum laude) and a B.A. in Math, Computer Science and Psychology. She has over 30 years of practical experience as a Project Manager and Information Systems Analyst.

Lior Haiman holds a B.Sc in Information Systems from The Academic College of Tel Aviv Yaffo. She has experience in information security and currently works as a Project Manager for "restart Group", which provides IT services and develops information systems \& websites. 